\title{
A friend in need: evaluating the impact of Lifebox in Burkina Faso
}

\author{
Angela Enright, MB, FRCPC
}

Received: 18 September 2018/Revised: 26 September 2018/Accepted: 26 September 2018/Published online: 27 November 2018 (c) Canadian Anesthesiologists' Society 2018

The letter from Bougouma et al. in this issue of the Journal $^{1}$ should be of great interest to readers, especially to members of the Canadian Anesthesiologists' Society (CAS). It outlines the impact of a gift from CAS members to colleagues in Burkina Faso.

In 2012, Professor Franco Carli, then Chair of the Board of the CAS International Education Foundation (CASIEF), was contacted by an anesthesia resident in Burkina Faso asking if the CAS could help with supplying training materials for their residency program. The Board of CASIEF responded by offering to provide 117 pulse oximeters. Additional oximeters (including pediatric oximetry probes) were donated by the Lifebox Foundation. ${ }^{2}$ Working with the World Federation of Societies of Anaesthesiologists (WFSA) and Lifebox, a team arrived in Burkina Faso in 2013 to deliver the oximeters and to provide the education necessary for their use.

Workshops were held at the Blaise Compaoré Hospital in Ouagadougou (the capital) and at the University Hospital in Bobo-Dioulasso, the second largest city in Burkina Faso. Our first task was to familiarize local faculty with the teaching materials as well as our teaching methods, which included small group sessions and handson workshops (which were all delivered in French). Together, we then ran the program for 118 participants who were mainly non-physician anesthesia providers. The course included an introduction to using the Surgical Safety Checklist (SSCL). ${ }^{3}$

\footnotetext{
A. Enright, MB, FRCPC $(\square)$

Department of Anesthesia, University of British Columbia, Royal Jubilee Hospital, 1952 Bay Street, Victoria, BC V8R 1J8, Canada

e-mail: acenright1@gmail.com
}

Dr. Bougouma's letter ${ }^{1}$ highlights the improvements in patient monitoring that followed. Almost one year after the workshops, he personally telephoned each of the in-charge anesthetists at the 57 hospitals that received oximeters. All the hospitals were systematically using them in the operating rooms and $94 \%$ of the hospitals were using them in their postanesthesia care units (PACU). Both CASIEF and Lifebox subsequently donated more oximeters specifically for the PACUs. What is more surprising is that $68 \%$ of the surveyed hospitals were using the SSCL some of the time and $23 \%$ were using it systematically. It is arguably easy to teach anesthesia providers to use an oximeter-the take-up is rapid because it makes their job easier. ${ }^{4}$ Nevertheless, it is a very different story when the whole surgical team is involved with the SSCL and the possible improved outcomes are not always immediately evident. ${ }^{5,6}$ Sixty-four percent of those Bougouma et al. ${ }^{1}$ surveyed suggested education of the whole surgical team. Accordingly, it is the intention of the CASIEF team to return to Burkina Faso to do just that, using a new program recently developed by a consortium of anesthesia, surgery, and nursing groups. It is called SAFE-OR and is currently in the pilot phase. SAFE is an acronym for Safer Anesthesia From Education, which includes SAFE-OB (Obstetric Anesthesia), SAFE-Peds (Pediatric Anesthesia), and now SAFE-OR (Operating Room). ${ }^{7}$

The simple interaction of asking for help and receiving it has had unexpected spin-off effects. It has led to the development of a long-term relationship between CASIEF and our colleagues in Burkina Faso. They have become part of the CASIEF family and several projects have grown from this. Professor N. Ouédraogo (President of the Société d'Anesthésie, de Réanimation et de Médecine d'Urgence du Burkina [SARMU-B]) was a guest speaker at the CAS annual meeting, giving members of CAS some insight into 
anesthesia practice in one of the poorest countries of the world. Dr. Bertille $\mathrm{Ki}$, one of their young pediatric anesthesiologists, is supported by CASIEF as part of a consortium devoted to the support and development of pediatric anesthesia and surgery in low- and middleincome countries; she has also been a guest at the University of Ottawa to improve her expertise in regional anesthesia. Dr. Cheik Bougouma, the lead author of the oximeter study outlined in the letter published in this issue, ${ }^{1}$ was supported by CASIEF to attend and present at the World Congress of Anaesthesiologists in Hong Kong in 2016. These are important efforts in the development of anesthesia in Burkina Faso.

In addition, CASIEF and the WFSA have returned to Burkina Faso to provide training in obstetric anesthesia by way of a SAFE-OB course. ${ }^{7,8}$ Thirty-two providers participated and 15 teachers were trained to deliver the course. All of the equipment necessary to run the course was donated to the SARMU-B allowing them to remain equipped to continue training their colleagues. Unfortunately, organizing these courses and getting government support to run them is difficult. Thus, they have not yet been able to develop this training project. Hopefully, this will come in time.

I believe that the relationship between CASIEF and the SARMU-B exemplifies some of the best in north-south relationships-i.e., SARMU-B asks for what help it needs, and CASIEF offers what support it can. This support has given our colleagues confidence that they can progress and improve anesthesia and surgery in their own country. For example, they have developed a day surgery program for children, which, to my knowledge, was the first in subSaharan Africa. ${ }^{9}$ They have also run a SAFE-Peds ${ }^{7}$ course in Ouagadougou. The CASIEF is in the planning stages for a multi-professional course on Essential Pain Management,${ }^{10}$ for which a grant has been received from the Louise and Alan Edwards Foundation in Montreal. Colleagues from Burkina Faso have also been invited to team-teach with CASIEF participants in other countries in the region; these efforts have been very well received. Unfortunately, our efforts have been hampered by the terrorist attacks that were visited upon Burkina Faso from nearby countries. ${ }^{11}$

Can we prove that all of these efforts have helped improve surgical and anesthesia care? No we cannot. Data are very difficult to obtain and Burkina Faso is one of the poorest countries in the world $-183^{\text {rd }}$ out of 189 on the human development index scale, with a maternal mortality ratio of 371/100,000 live births and a neonatal mortality of 25.6/1000 live births. ${ }^{12,13}$ Bad as these numbers may be, they are a significant improvement from those in years past. ${ }^{12,13}$ If the CAS has had a small part to play in this improvement, we should be very pleased and we should continue our efforts to assist our colleagues in Burkina Faso to make anesthesia safer everywhere.

\section{Un ami dans le besoin : évaluer l'impact du Lifebox au Burkina Faso}

La lettre de Bougouma et coll. publiée dans ce numéro du Journal $^{1}$ devrait grandement intéresser les lecteurs, et plus particulièrement les membres de la Société canadienne des anesthésiologistes (SCA). Elle souligne l'impact d'un cadeau des membres de la SCA à leurs collègues du Burkina Faso.

En 2012, le professeur Franco Carli, président du Conseil d'administration de la Fondation d'éducation internationale de la SCA (CASIEF) a été contacté par un résident en anesthésie du Burkina Faso demandant si la SCA pourrait contribuer à la fourniture de matériels de formation pour leur programme de résidence. Le Conseil d'administration de la CASIEF a répondu en offrant la fourniture de 117 oxymètres de pouls. Des oxymètres supplémentaires (incluant des capteurs pour oxymétrie pédiatrique) ont été données par la Lifebox Fondation. ${ }^{2}$ Travaillant avec la Fédération mondiale des sociétés d'anesthésiologie (FMSA) et Lifebox, une équipe est arrivée au Burkina Faso en 2013 pour livrer les oxymètres et assurer la formation nécessaire pour leur utilisation.

Des ateliers se sont déroulés à l'hôpital Blaise Compaoré, dans la capitale Ouagadougou, et à l'hôpital universitaire de Bobo-Dioulasso, la deuxième plus grande ville du pays. Notre première tâche a été de familiariser les enseignants locaux avec les matériels et nos méthodes d'enseignement qui incluaient des sessions en petits groupes et des ateliers pratiques (tous délivrés en français). Ensemble, nous avons mené ce programme à terme pour 118 participants qui étaient principalement des prestataires d'anesthésie non-médecins. Le cours a inclus une introduction à l'utilisation de la Liste de contrôle de sécurité chirurgicale (SSCL). ${ }^{3}$

La lettre du $\mathrm{D}^{\mathrm{r}}$ Bougouma ${ }^{1}$ souligne les améliorations dans le monitorage des patients qui ont suivi. Presque un an après les ateliers, il a personnellement téléphoné à chacun des anesthésistes responsables dans les 57 hôpitaux qui avaient reçu des oxymètres. Tous les hôpitaux les utilisaient de manière systématique en salle d'opération et $94 \%$ les utilisaient dans leurs unités de soins post anesthésiques (salle de réveil). La CASIEF et Lifebox ont ensuite donné d'autres oxymètres, spécifiquement pour les salles de réveil. Ce qui est le plus surprenant, c'est que 68 \% des hôpitaux interrogés utilisaient la SSCL de temps 
à autre et $23 \%$ l'utilisaient systématiquement. Il est théoriquement facile d'apprendre à un prestataire d'anesthésie comment utiliser un oxymètre: le changement est rapide parce que cela facilite leur travail. ${ }^{4}$ Néanmoins, c'est une toute autre histoire quand toute l'équipe chirurgicale est concernée par la SSCL et que l'amélioration possible de l'évolution des patients n'est pas toujours immédiatement évidente. ${ }^{5,6}$ Soixante-quatre pour cent de ceux qui ont été questionnés par Bougouma $e t$ coll. ${ }^{1}$ ont suggéré l'éducation de toute l'équipe chirurgicale. L'équipe de la CASIEF a donc l'intention de retourner au Burkina Faso pour ne faire que cela, en utilisant un nouveau programme récemment élaboré par un consortium de groupes d'anesthésistes, de chirurgiens et d'infirmiers. Il est appelé SAFE-OR et en est actuellement à la phase pilote. SAFE est l'acronyme pour «Safer Anesthesia From Education » (anesthésie plus sécuritaire grâce à l'éducation), ce qui inclut SAFE-OB (pour l'anesthésie obstétricale), SAFE-Peds (pour l'anesthésie pédiatrique) et maintenant SAFE-OR (pour la salle d'opération). ${ }^{7}$

La simple interaction consistant à demander de l'aide et à en recevoir a eu des conséquences inattendues. Elle a conduit au développement d'une relation à long terme entre la CASIEF et nos collègues du Burkina Faso. Ils sont entrés dans la famille de la CASIEF et plusieurs projets en sont issus. Le professeur N. Ouédraogo (président de la Société d'Anesthésie, de Réanimation et de Médecine d'Urgence du Burkina [SARMU-B]) était un conférencier invité du congrès annuel de la SCA et a expliqué à ses membres quelques notions des pratiques anesthésiques dans l'un des pays les plus pauvres du monde. La docteure Bertille Ki, l'une de leurs jeunes anesthésiologistes pédiatriques, bénéficie du soutien de la CASIEF dans le cadre d'un consortium consacré au soutien et au développement de l'anesthésie et de la chirurgie pédiatriques dans les pays à revenu intermédiaire et faible; elle a aussi été invitée par l'université d'Ottawa pour améliorer son expertise en anesthésie régionale. Le docteur Cheik Bougouma, le principal auteur de l'étude sur les oxymètres mise en avant dans la lettre publiée dans ce numéro, ${ }^{1}$ était soutenue par la CASIEF pour participer au Congrès mondial des anesthésiologistes à Hong-Kong en 2016 et pour y faire une présentation. Ce sont des efforts importants pour le développement de l'anesthésie au Burkina Faso.

De plus, la CASIEF et la FMSA sont revenus au Burkina Faso pour assurer une formation en anesthésie obstétricale à l'aide du cours SAFE-OB. ${ }^{7,8}$ Trente-deux prestataires ont participé et 15 enseignants ont été formés pour assurer le cours. Tout l'équipement nécessaire au cours a été donné au SARMU-B pour lui permettre de rester équipé et de continuer à former leurs collègues. Malheureusement, ces cours sont difficiles à organiser et il est aussi difficile d'obtenir un soutien gouvernemental pour les donner. En fait, ils n'ont pas encore pu développer ce projet de formation, mais on peut espérer que cela va se faire bientôt.

Je crois que les relations entre la CASIEF et le SARMU-B sont l'exemple même de certaines des meilleures relations Nord-Sud, c'est-à-dire que le SARMU-B demande l'aide dont il a besoin et la CASIEF offre le soutien qu'elle peut apporter. Ce soutien a donné confiance à nos collègues : ils peuvent progresser et améliorer l'anesthésie et la chirurgie dans leur propre pays. Ils ont, par exemple, élaboré un projet de chirurgie d'un jour pour les enfants qui, à ma connaissance, était le premier en Afrique subsaharienne. ${ }^{9}$ Ils ont également donné une série de cours SAFE-Peds ${ }^{7}$ à Ouagadougou. La CASIEF est en train de préparer un cours destiné à différents professionnels sur la Gestion essentielle de la douleur, ${ }^{10}$ pour lequel elle a reçu une bourse de la Fondation Louise et Alan Edwards de Montréal. Des collègues du Burkina Faso ont été également invités à se joindre à l'équipe enseignante avec les participants de la CASIEF dans d'autres pays de la région; ces efforts ont reçu un très bon accueil. Hélas, nos efforts ont été contrecarrés par des attaques terroristes qui se sont abattues sur le Burkina Faso depuis des pays voisins. ${ }^{11}$

Pouvons-nous prouver que tous ces efforts ont contribué à améliorer les soins chirurgicaux et anesthésiques? Nous ne le pouvons pas. Les données sont très difficiles à obtenir et le Burkina Faso est l'un des pays les plus pauvres du monde : $183^{\mathrm{e}}$ sur 189 sur l'échelle d'Indice du développement humain avec une mortalité maternelle de 371/100 000 naissances vivantes et une mortalité néonatale de 25,6/1000 naissances vivantes. ${ }^{12,13}$ Aussi mauvais que soient ces chiffres, ils montrent une amélioration significative par rapport aux années précédentes. ${ }^{12,13} \mathrm{Si}$ la SCA a eu un petit rôle à jouer dans cette amélioration, nous devrions en être très heureux et poursuivre nos efforts pour aider nos collègues du Burkina Faso à rendre l'anesthésie plus sécuritaire partout.

Conflict of interest Dr. Enright is a past President of WFSA, a past Chair of CASIEF, and is a current member of the Board of the Lifebox Foundation.

Editorial responsibility This submission was handled by Dr. Hilary P. Grocott, Editor-in-Chief, Canadian Journal of Anesthesia.

Funding None declared.

Conflit d'intérêts La $\mathrm{D}^{\mathrm{re}}$ Enright est ancienne présidente de la FMSA, ancienne présidente de la CASIEF et actuelle membre du Conseil d'administration de la Lifebox Fondation.

Responsabilité éditoriale Cet article a été traité par le $\mathrm{D}^{\mathrm{r}}$ Hilary $\mathrm{P}$. Grocott, Rédacteur en chef, Journal canadien d'anesthésie.

Financement Aucun déclaré. 


\section{References}

1. Bougouma CT, Ouédraogo $N$, Kaboré RA, et al. Évaluation de l'impact du projet Lifebox au Burkina Faso sur la pratique de l'oxymétrie pulsée et la check-list de l'organisation mondiale de la santé pour la sécurité du patient au bloc opératoire. Can J Anesth 2018; 65: DOI: https://doi.org/10.1007/s12630-018-1263-3.

2. Lifebox Foundation. Available from URL: https://www.lifebox. org (accessed September 2018).

3. Haynes $A B$, Weiser $T G$, Berry WR, et al. A surgical safety checklist to reduce morbidity and mortality in a global population. N Engl J Med 2009; 360: 491-9.

4. Finch LC, Kim RY, Ttendo S, et al. Evaluation of a large-scale donation of Lifebox pulse oximeters to non-physician anaesthetists in Uganda. Anaesthesia 2014; 69: 445-51.

5. Vivekanantham $S$, Ravindran $R P$, Shanmugarajah $K$, Maruthappu M, Shalhoub J. Surgical safety checklists in developing countries. Int J Surg 2014; 12: 2-6.

6. Urbach DR, Govindarajan A, Saskin R, Wilton AS, Baxter NN. Introduction of surgical safety checklists in Ontario. Canada. N Engl J Med 2014; 370: 1029-38.

7. World Federation of Societies of Anaesthesiologists. Safer Anaesthesia From Education (SAFE). Available from URL: https://www.wfsahq.org/wfsa-safer-anaesthesia-from-educationsafe (accessed September 2018).
8. Livingston P, Evans $F$, Nsereko E, et al. Safer obstetric anesthesia through education and mentorship: a model for knowledge translation in Rwanda. Can J Anesth 2014; 61: 1028-39.

9. Kabré YB, Traoré IS, Kaboré FA, et al. Anesthesia for ambulatory pediatric surgery in sub-Saharan Africa: a pilot study in Burkina Faso. Anesth Analg 2017; 124: 623-6.

10. Australian and New Zealand College of Anaesthetists; Faculty of Pain Medicine ANZCA. Essential Pain Management. Available from URL: https://wfsahq.org/images/EPM_Flyer_A4_ 20141219.pdf (accessed September 2018).

11. France Diplomatie. Burkina Faso - Terrorist Attack (16 June 2018). Available from URL: https://www.diplomatie.gouv.fr/en/ country-files/burkina-faso/events/article/burkina-faso-terroristattacks-16-06-18 (accessed September 2018).

12. World Health Organization. Maternal mortality in 1990-2015. WHO, UNICEF, UNFPA, World Bank Group, and United Nations Population Division Maternal Mortality Estimation Inter-Agency Group -Burkina Faso. Available from URL: http://origin.who.int/gho/maternal_health/countries/bfa.pdf (accessed September 2018).

13. World Data Atlas. Burkina Faso. Neonatal mortality rate. Available from URL: https://knoema.com/atlas/Burkina-Faso/ Neonatal-mortality-rate (accessed September 2018). 